\title{
Análise do potencial de imagem TerraSAR-X para mapeamento temático no sudoeste da Amazônia brasileira
}

César Edwin GARCIA ${ }^{1}$, João Roberto dos SANTOS$^{1}$, José Claudio MURA ${ }^{1}$, Hermann Johann Henrich KUX ${ }^{1}$

\section{RESUMO}

O presente trabalho tem como objetivo analisar o potencial de imagens SAR polarimétricas do sensor TerraSAR-X, no modo StripMap, para mapear o uso e cobertura da terra na região sudoeste da Amazônia brasileira. No procedimento metodológico imagens de amplitude nas polarizaçóes $\mathrm{A}_{\mathrm{HH}}$ e $\mathrm{A}_{\mathrm{VW}}, \mathrm{A}_{<\mathrm{HH} . \mathrm{VV}^{*}>}$ derivada da matriz de covariância, bem como da entropia $\mathrm{A}_{\text {Entropia }}$ derivada da decomposiçáo de alvos por auto-valores fizeram parte, de forma individual ou combinada, do conjunto de dados investigados. $\mathrm{Na}$ classificaçáo das imagens foram empregados dois classificadores: um baseado nas funçóes estatísticas de máxima verossimilhança (MAXVER); e outro, o método contextual (Context). Os resultados temáticos dessas classificaçóes foram avaliados através da matriz de confusão e pelo índice Kappa. De forma sintetizada pode-se afirmar que as componentes $\mathrm{A}_{<\mathrm{HH.VV^{* }}}$ e $\mathrm{A}_{\text {Entropia }}$ têm significativa contribuição no procedimento classificatório, sobretudo pelo método Context, cujo desempenho alcançou com $78 \%$ de exatidão global e índice Kappa de 0,70 .

PALAVRAS-CHAVE: mapeamento florestal, radar, classificação polarimétrica, Amazônia.

\section{Analysis of the potential use from TerraSAR-X images for thematic mapping in SW Brazilian Amazon region}

\section{ABSTRACT}

The objective of this work was to analyze the potential use of SAR polarimetric images from the TerraSAR-X sensor system, at StripMap mode, to map land use and land cover in SW Brazilian Amazon. Amplitude images at polarizations $\mathrm{A}_{\mathrm{HH}}, \mathrm{A}_{\mathrm{VW}}$, $\mathrm{A}_{<\mathrm{HH} . \mathrm{V} \mathrm{V}^{*}}$, derived from the co-variance matrix, as well as the entropy $\mathrm{A}_{\text {Entropia }}$, derived from the decomposition of targets by eigenvalues, are parts of the datasets investigated individually or in combined form. Two classifiers were used: the first is based on statistical functions of maximum likelihood (MAXVER), and the second is the contextual method (Context). The thematic results from these classifications were evaluated by a confusion matrix and by the Kappa index. Summarizing we can state that the components $\mathrm{A}_{<{\mathrm{HH} . \mathrm{VV}^{*}}^{*}}$ and $\mathrm{A}_{\text {Entropia }}$, gave a significant contribution to the image classification procedure, considering specially the Context method, whose performance reached 78\% of Global Accuracy and a Kappa index of 0.70.

KEYWORDS: Forest mapping, Radar, Polarimetric classification, Amazon. 


\section{INTRODUÇÃO}

O conhecimento da dinâmica de uso e cobertura da terra exerce papel importantíssimo para entender os fenômenos resultantes da atividade humana que ocorrem em áreas tropicais, especificamente na regiáo da Amazônia brasileira. A conversão da floresta em outras classes de uso da terra tem ocasionado excessiva fragmentaçáo de habitats, perda de biodiversidade, degradação de solos férteis e redução da capacidade hídrica em certas zonas amazônicas. Face esses desequilíbrios resultantes da ação humana nos ecossistemas amazônicos, torna-se necessário um constante monitoramento da paisagem, conforme reportado por Houghton et al. (2000) e Vieira et al. (2004). Produtos de sensores remotos, em nível orbital, têm sido ferramentas adequadas para auxiliar no entendimento e monitoramento dessas áreas. Não obstante, sensores que operam na faixa óptica mostram limitaçóes na aquisição regular de dados, devido à constante e elevada cobertura por nuvens no decorrer do imageamento, sobretudo em certos setores amazônicos, conforme citado por Asner (2001). Por conseguinte, imagens dos radares de abertura sintética (SAR) são, de maneira relativa quanto a operacionalidade do programa de imageamento, alternativas viáveis, devido a independência da aquisição em relaçáo às condições atmosféricas das regióes tropicais (Henderson e Lewis 1998).

Há disponibilização de imagens SAR oriundas de diferentes freqüências e polarizaçôes, podendo-se citar: imagens do Advanced Land Observing Satellite (ALOS/ PALSAR, banda L); do Radarsat-2 (banda C); do TerraSAR (banda X); e da constelação COSMO-SkyMed (banda X). $\mathrm{O}$ crescente interesse da comunidade técnico-científica em aprimorar ferramentas de tratamento desses dados SAR tem demonstrado suas aplicabilidades em: mapeamento do uso e cobertura da terra (Santos et al. 2008; Freitas et al. 2008); diferenciação entre estádios de sucessão florestal (Rignot et al 1997; Neeff et al. 2005a); discriminação entre áreas de floresta primária, áreas desmatadas e de corte seletivo (Van der Sanden e Hoekman 1999; Saatchi et al. 1997); caracterizaçáo de respostas polarimétricas de florestas primárias e secundárias (Santos et al. 2009); estimativa de parâmetros biofísicos da tipologia florestal e sua biomassa (Kasischke et al. 1997; Hoekman e Quiñones 2000; Santos et al. 2002; Narvaes 2010).

Os sensores-radar apresentam algumas restriçôes quanto à utilidade para determinadas aplicaçóes, de acordo com as características intrínsecas de operação, em razão da freqüência e da polarização utilizada. No caso de estudos florestais, é importante conhecer qual é a frequencia em que opera o radar e, por conseguinte, a capacidade de interaçáo do sinal emitido com a estrutura da floresta (troncos, galhos e folhas). O comprimento de onda, por exemplo, é o parâmetro de maior influência na capacidade de penetração do sinal de microondas no dossel florestal. Na banda X $(2,4 \mathrm{~cm}-3,75 \mathrm{~cm})$, o pequeno comprimento de onda, praticamente náo penetra no dossel e, portanto, o retroespalhamento ocorre na superfície, com as folhas e pequenos galhos formadores do dossel. Na banda C $(3,75 \mathrm{~cm}-7,5 \mathrm{~cm})$ a penetração é um pouco maior e a interação se dá com folhas e galhos maiores formadores da copa das árvores do estrato superior. Nos comprimentos de onda maiores, caso das bandas L $(15 \mathrm{~cm}-30 \mathrm{~cm})$ e P $(77 \mathrm{~cm}$ $-136 \mathrm{~cm}$ ), dependendo da densidade, estrutura da vegetação e umidade, o sinal de microondas emitido penetra o dossel, interage com toda e variada estrutura florestal e pode chegar até o solo (Leckie e Ranson 1998).

Dentro desse contexto, visando explorar a potencialidade de novos sensores SAR, o objetivo da presente pesquisa foi avaliar a aplicabilidade de imagens polarimétricas dual (HH e VV) do TerraSAR-X, no modo StripMAP para a identificaçáo de classes de cobertura e uso da terra na porção SW da Amazônia brasileira. Para tal, técnicas de tratamento POLSAR, como a decomposição de alvos e de classificaçóes, contextual e estatístico por máxima verossimilhança, foram empregadas para o mapeamento temático da regiáo estudada.

\section{Síntese teórica dos conceitos SAR e suas aplicações em estudos da tipologia florestal}

A base teórica sobre as interaçóes radar versus alvo foi convenientemente estabelecida a partir da década 80 (Ulaby et al. 1981; Ulaby e Dobson 1989). No caso específico da cobertura florestal, esta é representada numa imagem SAR, segundo Leckie e Ranson (1998), pela combinação das respostas geradas através dos diferentes mecanismos de espalhamento. Essas incluem a contribuição direta do solo, do tronco, da copa das árvores, da interação copa-solo e da reflexão dupla tronco-solo. A magnitude dos mecanismos de espalhamento decorrentes dos diferentes componentes é dependente dos fatores geométricos e das propriedades dielétricas da vegetação e do solo (Dobson et al. 1995). Comprimento de onda, polarização e ângulo de incidência do sinal controlam os mecanismos de espalhamento múltiplo, superficial e/ou volumétrico que expressam a resposta polarimétrica de alvos florestais.

Um radar polarimétrico permite extrair informaçóes referentes à textura e ao brilho, obtidos a partir da magnitude, mas também, possibilita caracterizar os mecanismos de espalhamento do alvo. As características polarimétricas de um determinado alvo podem ser descritas por uma série de atributos extraídos da matriz de espalhamento complexa [S] (amplitude e fase), que constitui uma matriz [2×2] de dados complexos, contendo informaçóes relativas ao alvo (Ulaby e Elachi 1990). Estes atributos podem ser divididos em "incoerentes", referentes às medidas de potência do radar, e 
"coerentes", que fazem uso da informação de fase (Henderson e Lewis 1998). Entre os atributos incoerentes podem ser citados o coeficiente de retroespalhamento $\left(\sigma_{0}\right)$, a razão de polarização paralela $(\mathrm{Rp})$, a razão de polarização cruzada $(\mathrm{Rc})$, a potência total (PT) e os índices biofísicos desenvolvidos por Pope et al. (1994), os quais visam aumentar a capacidade de caracterização de parâmetros florestais e o aproveitamento das diferentes polarizaçóes adquiridas. Entre os atributos coerentes estão a diferença de fase $(\Delta \Phi)$, a coerência polarimétrica $(\gamma)$ entre as polarizaçóes $\mathrm{HH}$ e $\mathrm{VV}$, a entropia $(\mathrm{H})$, a anisotropia (A) e o ângulo alfa médio, resultantes da decomposição por autovetores da matriz de coerência (Cloude e Pottier 1997), a magnitude ( $\alpha s)$ e a fase de Touzi ( $\Phi \alpha s)$, ambas derivadas da decomposição por autovalor e autovetor da matriz de coerência [T], além do ângulo de orientação $(\psi)$ e heliticidade $(\chi)$ e as componentes de espalhamento volumétrico $(\mathrm{Pv})$, double bounce (Pd) e superficial (Ps), resultantes da decomposição de Freeman (Freeman e Durden 1998), na matriz de covariância [C].

A partir da matriz de espalhamento $[S]$ pode-se gerar a matriz de covariância [C] e de coerência [T]. As matrizes de covariância e de coerência são unitariamente similares, carregando as mesmas informaçóes, tendo os mesmos autovalores (que são reais), mas com diferentes autovetores (Cloude e Pottier 1996). A matriz de covariância ou de coerência corresponde a parâmetros de entrada para a decomposição por autovalor e autovetor (Cloude e Pottier 1997). Com base nesta decomposição obtém-se uma interpretação física dos mecanismos de espalhamento, através do conceito de entropia, anisotropia e do ângulo $\alpha$. A Entropia (H) mede a quantidade de aleatoriedade (ou desordem) dos processos de espalhamento, o ângulo $\alpha$ é utilizado para descrever o tipo de mecanismo que rege o espalhamento e a Anisotropia (A) permite estimar a importância relativa dos diferentes mecanismos de espalhamento (Cloude e Pottier 1997).

A aplicabilidade dos dados SAR, quanto à relação e análise dos seus diversos atributos, decomposição de alvos e algoritmos classificatórios polarimétricos, tem um vasto campo científico: desde a questão de caracterização da tipologia florestal e mapeamento temático (Van der Sanden e Hoekman 1999; Santos et al. 2008); a questão de análise de respostas polarimétricas de alvos florestais (Mc Nairn et al. 2002; Santos et al. 2009); os estudos de parâmetros biofísicos para a modelagem volumétrica e de biomassa (Santos et al. 2002; Neeff et al. 2005a; Neeff et al. 2005b; Saatchi et al. 2007; Gonçalves et al. 2011); até o uso específico de classificadores polarimétricos no delineamento de classes de uso e cobertura da terra, como os trabalhos de: Freitas et al. (2008) e Guerra et al. (2010) ao fazerem uso do Iterated Conditional Mode - ICM; Cloude e Pottier (1997) ao empregarem atributos coerentes no plano $\mathrm{H} / \alpha$; e Rodrigues (2010) e Lim et al. (2008) ao utilizarem informação contextual.

\section{MATERIAL E MÉTODOS}

A área investigada está localizada no Município de Sena Madureira, Estado do Acre, entre os paralelos 9 '00' e 945' $S$ e meridianos 68 $60^{\circ}$ e $68^{\circ} 35^{\prime} \mathrm{W}$ (Figura 1). A formação vegetal predominante na região é a Floresta Ombrófila Aberta Submontana, com ocorrência ainda de Floresta Ombrófila Densa Aluvial com dossel uniforme. Face ao processo de conversão da floresta em atividades agropecuárias, ocorrem áreas de sucessão secundária, formadas pelo abandono de uso tradicional com o esgotamento da fertilidade do solo. O relevo é dissecado de topo tabular, com densidade de drenagem média e baixo grau de entalhamento dos canais (RADAMBRASIL 1978). As precipitaçóes mensais mais baixas na regiāo ocorrem em Junho e Agosto $(50 \mathrm{~mm})$, e as mais altas $(270 \mathrm{~mm})$ nos meses de Dezembro e Fevereiro (CPTEC 2010).

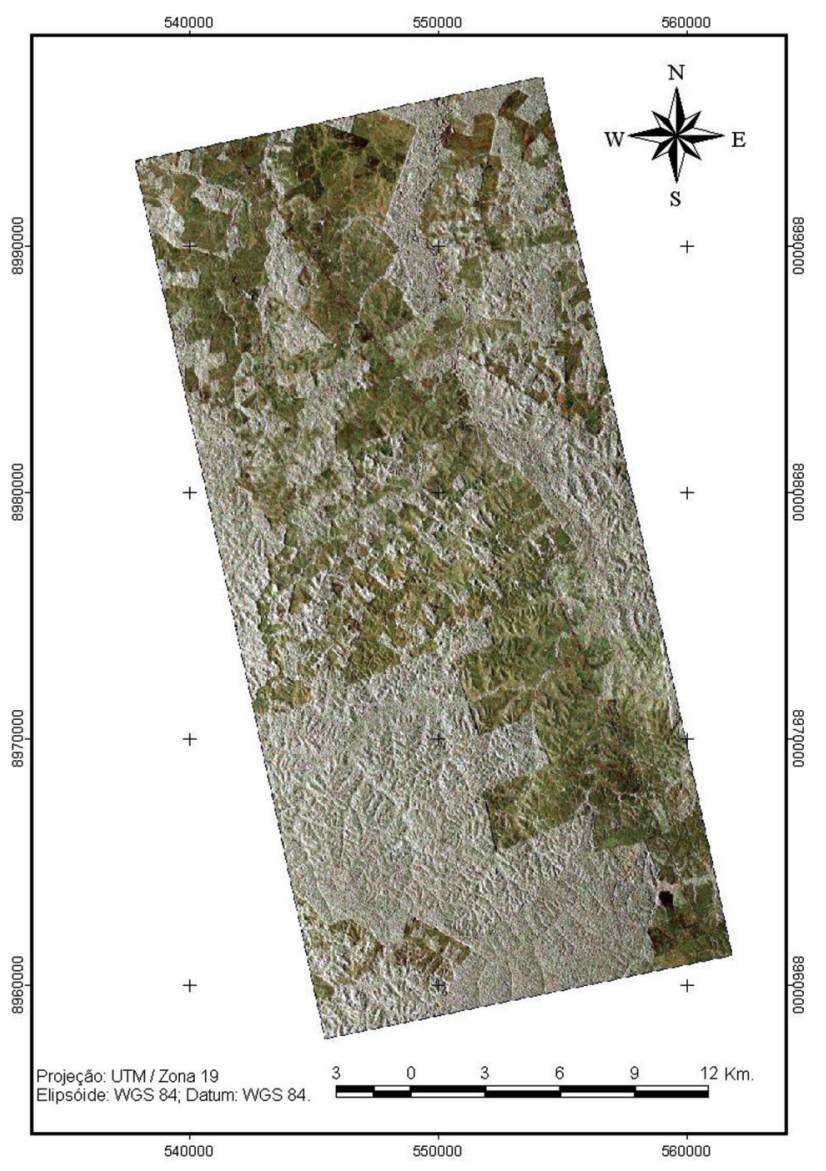

Figura 1 - Localização da área no município Sena Madureira (AC) observado em imagem TerraSAR-X. 
No presente estudo empregou-se uma imagem SAR polarimétrica do TerraSAR-X (banda X) (Werninghaus e Buckreuss 2010; Pitz e Miller 2010), originalmente em formato Single Look Complex - SLC (Henderson e Lewis 1998), no modo de operação StripMap (Eineder et al. 2008), com passagem ascendente e data de imageamento realizada em 24/06/2009. Dentre as características dessa imagem SAR trabalhada têm-se: polarizações $\mathrm{HH}$ e VV, com resolução espacial de $1,124 \mathrm{~m}$ (Range) e 6,59 m (azimute), ângulo de incidência de $43,46^{\circ}$ (near) e $44.61^{\circ}$ (far) e faixa imageada de $36 \mathrm{~km}$ (azimute) e 16,6 km(range).

$\mathrm{Na}$ Figura 2a pode-se observar, de forma sintetizada, o fluxograma metodológico geral adotado nesse trabalho. Em uma primeira fase do trabalho constam as funçóes de préprocessamento dos dados TerraSAR-X (Figura 2b), onde se gerou a matriz de covariância, efetuou-se a decomposiçâa de alvos e a geração das imagens-componentes para serem utilizadas no processo classificatório. Posteriormente foi realizada uma ortorretificação da imagem, seguida das classificaçôes propriamente ditas, com as respectivas validaçôes temáticas.

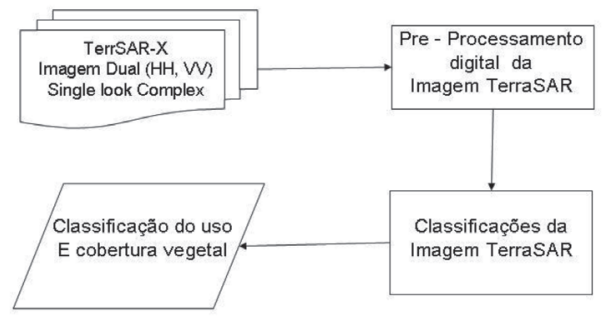

(a)

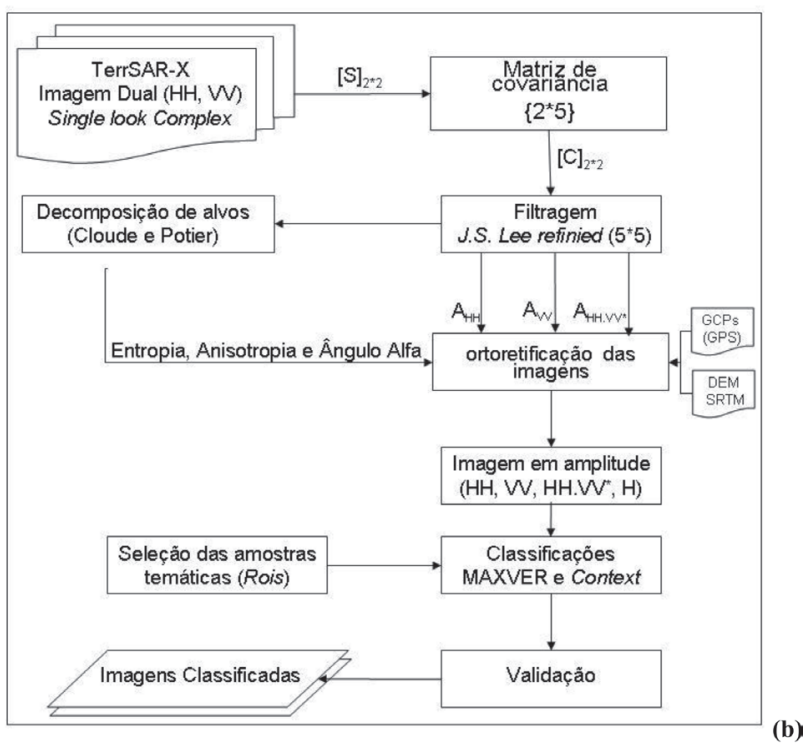

Figura 2 - Diagrama com a organização geral do trabalho (a) e procedimentos adotados nas etapas de pré-processamento e classificação (b).
A imagem TerraSAR-X foi processada no aplicativo PolsarPro 4.02 (http://earth.esa.int/polsarpro/install.html), gerando-se inicialmente a matriz de espalhamento $[S]_{2{ }_{2}}$ - Como os radares de polarizaçáo dual não permitem a reconstruçáo da matriz de espalhamento $[S]$ completa, pode-se reconstruir uma coluna da matriz, gerando uma nova banda sintética (Cloude 2007). Isso é feito a partir das informaçóes de fase das polarizaçóes $\mathrm{HH}$ e VV da imagem original, obtendo-se assim, a componente HH.VV*.

A matriz de covariância foi gerada aplicando-se uma média espacial e reamostragem de $2 \times 5$ sobre os elementos da matriz [S] para que o espaçamento entre pixels na direçấo range e azimute ficassem aproximadamente iguais. Com o objetivo de reduzir o ruído speckle, aplicou-se uma filtragem nos elementos da matriz $\left[\mathrm{C}_{2}\right],\left(\mathrm{C}_{\mathrm{HH}}, \mathrm{C}_{\mathrm{VV}}\right.$ e $\left.\mathrm{C}_{<\mathrm{HH} . \mathrm{VV}}\right)$. Inicialmente foram testados alguns filtros (Lee, Lee refinied, Frost) com diferentes tamanhos de janelas $(3 \times 3,5 \times 5$ e $7 \times 7)$. Considerando o desempenho dos mesmos, sem comprometer a resoluçáo espacial final, foi então escolhido o filtro J.S. Lee refinied (Lee et al. 1999), com tamanho de janela igual a $5 \times 5$.

A partir da matriz de covariância $\left[\mathrm{C}_{2}\right]$ filtrada, foram extraídos os parâmetros entropia $(\mathrm{H})$, anisotropia $(\mathrm{A})$, ângulo alfa $(\alpha)$ e o ângulo Beta $(\beta)$ derivados da decomposiçáo de alvos por autovalores e autovetores, utilizando o aplicativo PolsarPRO. É importante mencionar que a base conceitual da decomposição de alvos foi desenvolvida para dados SAR QuadPol (Cloude e Pottier 1997), podendo entretanto ser aplicada para dados de polarizaçáo dual (Cloude 2007), conforme anteriormente mencionado.

Os elementos da matriz de covariância $\left(\mathrm{C}_{\mathrm{HH}}, \mathrm{C}_{\mathrm{VV}} \mathrm{e}\right.$ $\mathrm{C}_{<{\mathrm{HH} . \mathrm{VV}^{*}}^{*}}$, que representam dados em intensidade, foram transformados em dados de amplitude $\left(\mathrm{A}_{\mathrm{HH}}, \mathrm{A}_{\mathrm{VV}}\right.$ e $\mathrm{A}_{<\mathrm{HH}}$. $\left.\mathrm{vV}^{*}\right)$ aplicando-se a raiz quadrada desses elementos. Depois dos processos de média, filtragem e transformação para amplitude, esses dados através de uma análise de histogramas foram avaliados e considerados como tendo distribuição do tipo gaussiana, podendo então ser utilizados como imagens de entrada no uso de classificador baseado em máxima verossimilhança.

Para que as classes temáticas pudessem ser relacionadas espacialmente com os dados gerorreferenciados de campo, o conjunto de imagens-radar em amplitude foi ortorretificado, utilizando o módulo OrthoEngine do software PCI Geomatics 10.3. A modelagem escolhida foi o método Radar Satellite Modelling, o qual utiliza ao máximo as informaçôes de efemérides do satélite, necessitando apenas de um número mínimo de 8 pontos de controle (GCPs) em conjunto com equaçôes de colinearidade e coplanaridade e de um modelo digital de elevação (DEM). O DEM empregado foi derivado dos dados SRTM3 (http://dds.cr.usgs.gov/srtm/version2_1/ SRTM3/South_America/). Como resultado foi obtida uma 
ortoimagem TerraSAR-X derivada dos dados originais, apresentando um valor de RMS (Root Mean Square) de 2,69 metros.

Como o objetivo principal da presente pesquisa foi avaliar o potencial do TerraSAR-X para discriminar classes de uso e cobertura do solo em áreas tropicais, adotaramse dois métodos de classificação: o primeiro, baseado em um classificador contextual (Context) e o segundo, em um classificador estatístico pontual por Máxima Verossimilhança - MAXVER.

A classificação contextual empregada fez uso do algoritmo de Gong e Howarth (1992), baseado na freqüência e no valor de cinza do vetor-redução para a identificação das classes temáticas de interesse. Este algoritmo foi utilizado por Rodrigues (2010) e por Lim et al. (2008) em imagens ALOS/ PALSAR em dados de amplitude (HH e HV) para mapear o uso do solo.

Por sua vez, o classificador MAXVER considera a ponderação das distâncias entre médias e a dependência espacial dos níveis digitais entre as classes, utilizando parâmetros estatísticos na classificação (Vieira 1996). Trabalhos empregando o classificador MAXVER com dados SAR foram realizados por: Rignot et al. (1997), ao discriminarem desflorestamentos e estágios de regeneração em floresta tropical; Saatchi et al. (1997) ao mapearem a cobertura do solo no Estado de Rondônia; Van Der Sanden e Hoekman (1999), no mapeamento de áreas florestais, de corte seletivo e de desflorestamento na Guiana e na Colômbia; Lee et al. (2001) ao discutirem a capacidade do classificador de Máxima Verossimilhança na discriminação do uso e cobertura da terra, utilizando dados polarimétricos full, dual e single, comparando-o ao classificador de Wishart.

Para utilizar o classificador contextual o primeiro passo foi aplicar uma conversão nas imagens amplitude de 32 bits para 8 bits, processo esse realizado através do algoritmo Scale (PCI Geomatics). O segundo passo foi a geração de uma imagem redução, no qual um conjunto de imagens é reduzido para uma imagem de uma dimensão, com perda relativamente pequena da informação. A redução foi então realizada usando-se o algoritmo Reduc (Gong e Howarth 1992). Neste processo foram testados vários conjuntos de produtos, tais como: a) todas as imagens, $\left(\mathrm{A}_{\mathrm{HH}}, \mathrm{A}_{\mathrm{vv}}(\mathrm{H}),(\mathrm{A})\right.$, ângulo alfa e $\left.\mathrm{A}_{<\mathrm{HH} . \mathrm{VV}^{*}}\right)$; b) conjunto $A_{H H}$ e $A_{v w}$ c) conjunto $A_{H H} A_{V V}$ e $\mathrm{H}, d$ ) conjunto $\mathrm{A}_{\mathrm{HH}}, \mathrm{A}_{\mathrm{V}}, \mathrm{H}$ e A. Nessa análise prévia verificou-se que o melhor resultado foi obtido com o conjunto $\left(A_{H H}, A_{V V}, A_{<H H . V V^{*}>} e H\right)$ e sobre esta imagem-redução aplicou-se o classificar contextual Context (Gong e Howarth 1992). Ao mesmo tempo dessa análise prévia, verificou-se que os atributos incoerentes de anisotropia (A) e ângulo alfa, bem como a informação de fase relativa contida no dado PolSAR, não forneceram ganho de informação suficiente para distinguir classes com significância desejada. Para a classificação MAXVER foi também usado o mesmo conjunto de imagens em amplitude $\left(\mathrm{A}_{\mathrm{HH}}, \mathrm{A}_{\mathrm{vv}}, \mathrm{A}_{<\mathrm{HH}}\right.$ $\mathrm{VV}^{*}$, e H) da utilizada na classificação Context.

As amostras de treinamento e validação empregadas nas classificaçôes foram selecionadas a partir dos dados levantados em campo. Esse trabalho de campo constou do inventário florestal de 12 transectos, com mensuração de variáveis dendométricas e de identificação botânica, o que permitiu a caracterização fisionômico-estrutural da tipologia florestal. Adicionalmente, foram coletados 189 pontos de observação georreferenciados com a descrição das unidades de uso e cobertura da terra. Com base nessas informaçóes foi estabelecida uma legenda com seis classes temáticas: floresta ombrófila aberta (primária), capoeira (sucessão secundária), pasto sujo, pasto limpo, solo exposto e corpos d' água.

Após a aplicação das duas abordagens classificatórias acima citadas, realizou-se uma fase de pós-classificação. Esta consistiu em aplicar um filtro de moda (9x9) para reduzir o aspecto ruidoso dos dados temáticos resultantes dessas classificaçóes.

Para analisar a separabilidade das classes foi calculada a distância estatística JM, conforme Richards e Jia (1999), medida essa efetiva para definir diferenças entre amostras representativas de determinados temas em imagens de satélite. Segundo este índice as amostras com maior semelhança apresentam valores próximos a zero (0); conseqüentemente, os valores próximo a dois (2) correspondem àquelas com maior diferenciação de resposta. Além disso, a partir de pontos-testes de observação definidos na etapa de campo foi realizada a validação do desempenho das classificaçôes, calculada através do grau de exatidão total e a exatidão por classes, decorrendo assim o índice de concordância Kappa (Landis e Koch 1977). Gráficos do tipo box-plot foram igualmente empregados para validar o desempenho do conjunto de imagens.

Por último, a partir dos valores de Kappa encontrados para cada classificação (MAXVER e Context), foi realizado um teste estatístico $Z$ para verificar, numa combinação dois a dois, se havia diferença significativa entre os resultados.

\section{RESULTADOS E DISCUSSÃO}

Na região sudoeste da Amazônia, uma agricultura de subsistência (para produção de arroz, mandioca, feijão, milho) ocupa geralmente áreas das pequenas propriedades rurais, juntamente com a criaçáo de pequeno lote de bovinos. Por outro lado, ao longo da estrada (BR 364) que liga Sena Madureira - Rio Branco e em suas vicinais, nota-se que houve forte conversão da floresta ombrófila aberta em extensivas áreas de pastagens, muitas delas em relevo dissecado de topo tabular. Âreas de floresta degradada por exploraçáo madeireira, de médio e baixo impacto, foram também observadas no 
decorrer do trabalho de campo, bem como um baixo número de áreas de sucessão secundária (capoeiras), decorrentes do abandono da área após tradicional uso agrícola e/ou pecuário e consequentemente, com perda da fertilidade do solo. Áreas de pastagem bem manejadas (pasto limpo) e outras com presença de espécies invasoras arbustivas e palmeiras espacialmente esparsas (pasto sujo) fazem parte da paisagem. O tema solo exposto identificado correspondia a áreas de agricultura em preparação para o plantio, ou em pousio.

O conhecimento fisionômico-estrutural e florístico é importante para os estudos da tipologia florestal com base em dados SAR, pois não somente permite categorizar cada tipologia florestal em estudo, como também entender a resposta polarimétrica e o mecanismo de espalhamento predominante entre o sinal-radar com o alvo florestal, conforme relatado por Santos et al. (2009). Por isso, foi executado o inventário florestal na presente pesquisa, com mensuração e identificação botânica de 1048 árvores (DAP> $10 \mathrm{~cm}$ em amostras de $1 / 4$ hectare para floresta e DAP > 5 $\mathrm{cm}$ em amostras de $1000 \mathrm{~m}^{2}$ para capoeira) cuja síntese de informaçóes está contida na Tabela 1.

Numa análise exploratória, pode-se observar na Figura 3 os gráficos do tipo box-plot que mostram o comportamento polarimétrico do conjunto de dados TerraSAR-X de cada classe temática, com as representaçóes dos parâmetros descritivos do primeiro e terceiro quartil, além da mediana. As polarizaçóes duais $\mathrm{HH}$ e VV, de maneira individualizada, mostraram-se limitadas para separar as diversas classes de uso e cobertura da terra. Levando em conta as componentes combinadas $<\mathrm{HH}$. $\mathrm{VV}^{*}>$ e Entropia $(\mathrm{H})$ nota-se uma maior diferenciação entre as classes.

Tabela 1 - Número total de indivíduos (N), espécies (s), famílias (F) e espécies de cada parcela amostral do IF, com os respectivos valores do índice de Shannon-Weaver $\left(H^{\prime}\right)$ e equabilidade de Pielou (J) para cada amostra florestal inventariada.

\begin{tabular}{cccccc}
\hline Parcela & N & S & F & H' & J \\
\hline Floresta 1 & 117 & 37 & 20 & 3,19 & 0,88 \\
\hline Floresta 2 & 60 & 32 & 24 & 3,27 & 0,94 \\
\hline Floresta 3 & 108 & 55 & 31 & 3,72 & 0,93 \\
\hline Floresta 4 & 115 & 50 & 26 & 3,6 & 0,92 \\
\hline Floresta 5 & 105 & 38 & 26 & 3,19 & 0,88 \\
\hline Floresta 6 & 132 & 35 & 21 & 2,85 & 0,8 \\
\hline Floresta 7 & 105 & 36 & 23 & 2,89 & 0,81 \\
\hline Floresta 8 & 123 & 39 & 22 & 3,15 & 0,86 \\
\hline Capoeira 1 & 40 & 24 & 21 & 2,94 & 0,92 \\
\hline Capoeira 2 & 36 & 11 & 11 & 1,79 & 0,75 \\
\hline Capoeira 3 & 36 & 16 & 14 & 2,38 & 0,86 \\
\hline Capoeira 4 & 71 & 15 & 11 & 1,85 & 0,68 \\
\hline Geral & 1048 & 136 & 50 & 4,1 & 0,84 \\
\hline
\end{tabular}

Fonte: Garcia 2011.

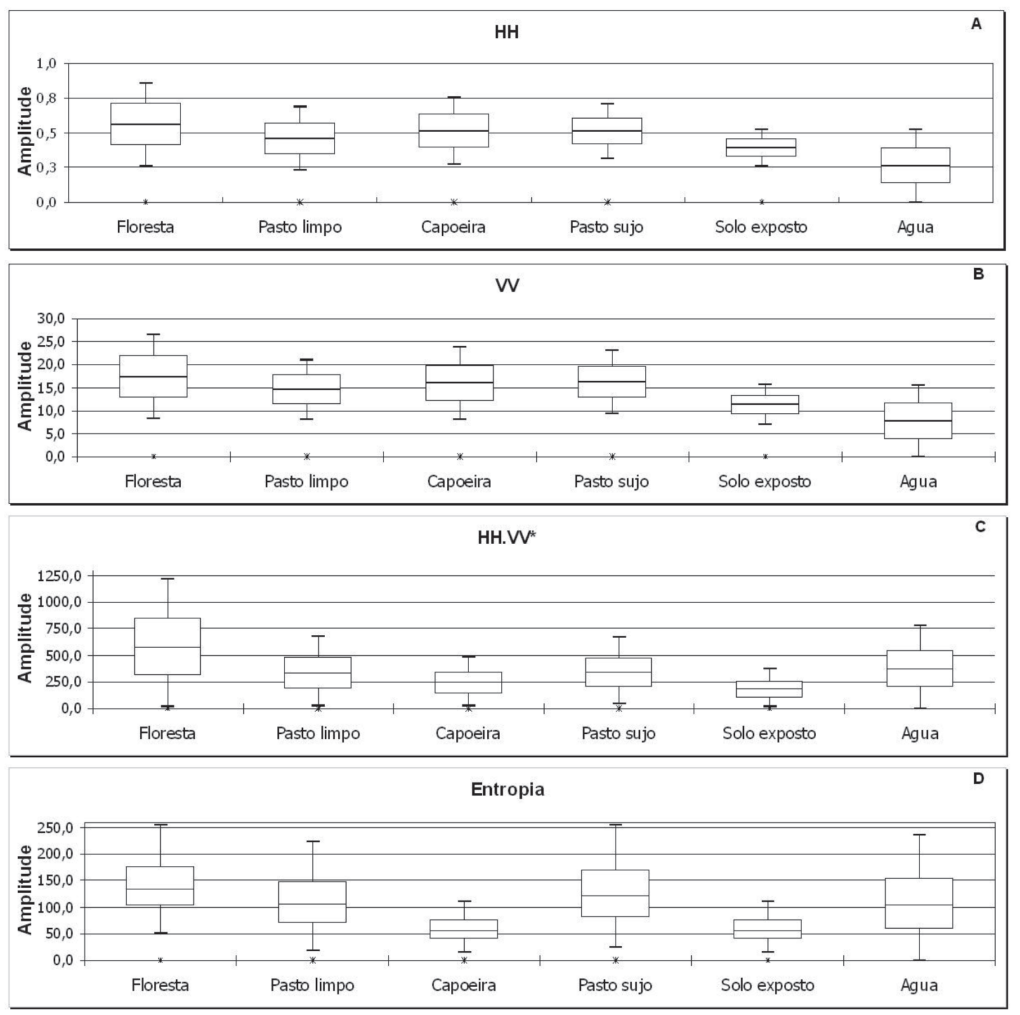

Figura 3 - Gráficos box-plot das classes de uso e cobertura da terra nas imagens TerraSAR em modos $A_{H H}$ (a), $A_{W W}\left(\right.$ b), $A_{<H H . W^{*}>}$ (c) e Entropia (d). 


\section{Classificação Maxver}

A classificação pelo método de máxima verossimilhança obteve uma boa concordância, segundo a categorização de Landis e Koch (1977), indicada pelo valor do índice Kappa encontrado de 0,53 . Na Figura 4 é apresentada a distribuição espacial das classes temáticas para essa região de Sena Madureira (AC), bem como a respectiva matriz de confusão e índice de concordância Kappa (Tabela 2), indicando o nível de desempenho desse classificador estatístico pontual.

Particularizando a discriminação entre classes, o melhor desempenho classificatório foi do tema solo exposto com $98 \%$, seguido pelo pasto limpo $81 \%$. A classe floresta apresentou apenas $55 \%$ dos pixels classificados corretamente, sendo que as classes capoeira (43\%) e pasto sujo (51\%) foram as que apresentam maior dificuldade de distinçáo, inclusive comprovado quando se calcula os valores da distância JeffriesMatusita $(J M=0,312)$ entre classes. Esta baixa separabilidade poder ser explicada pela complexidade polarimétrica apresentada pelas classes acima mencionadas, em razão,

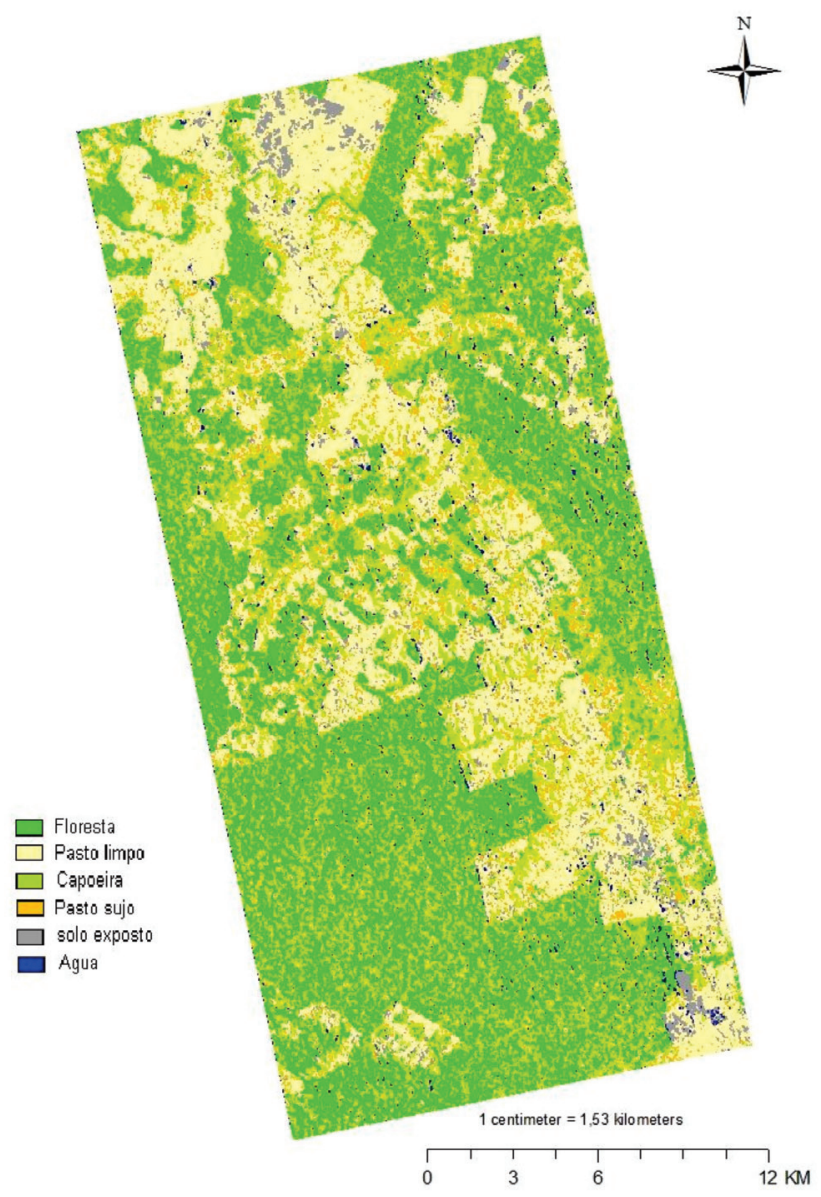

Figura 4 - Classificação temática pelo método MAXVER através do conjunto de imagens $\left(A_{H H}, A_{v^{\prime}} A_{<H н . W^{*}>}, A_{\text {entropia }}\right)$.
Tabela 2 - Matriz de confusão derivada da classificação MAXVER

\begin{tabular}{lcccccc}
\hline & Água & Capoeira & Floresta & $\begin{array}{c}\text { Pasto } \\
\text { limpo }\end{array}$ & $\begin{array}{c}\text { Pasto } \\
\text { sujo }\end{array}$ & $\begin{array}{c}\text { Solo } \\
\text { exposto }\end{array}$ \\
\hline Água & $\mathbf{3 2 , 0 3}$ & 0,02 & 0,33 & 0,33 & 0,00 & 0,00 \\
Capoeira & 2,57 & $\mathbf{4 3 , 7 4}$ & 25,10 & 3,42 & 17,57 & 0,00 \\
Floresta & 0,00 & 4,88 & $\mathbf{5 5 , 0 9}$ & 0,00 & 1,80 & 0,00 \\
Pasto limpo & 6,29 & 22,92 & 5,25 & $\mathbf{8 0 , 7 8}$ & 28,00 & 1,86 \\
Pasto sujo & 1,41 & 27,45 & 13,85 & 9,50 & $\mathbf{5 0 , 9 4}$ & 0,00 \\
Solo exposto & 57,70 & 1,00 & 0,37 & 5,97 & 1,69 & $\mathbf{9 8 , 1 4}$ \\
\hline
\end{tabular}

Exatidão Global $=63.70 \%$ Kappa $=0.530(\mathrm{Var}=5,43 \mathrm{E}-04)$

possivelmente, da ocorrência em áreas de relevo dissecado, com particular influência de sombras ou diferenças de iluminação, dependendo da variável geomorfométrica "pendente" na direção do imageamento desse SAR em banda X. Acrescentese a isso outros dois fatores: o primeiro, decorrente do fato de tratar essa complexidade polarimétrica das classes estabelecidas por um classificador pontual, de reconhecimento gaussiano, o que pode não estar devidamente adaptado às análises SAR para um conjunto de classes de concentrada distribuição de freqüências no espaço de atributos-radar que as definem; o segundo, decorrente do fato do sistema TerraSAR operar em banda $\mathrm{X}$, cuja frequência não permite maior penetrabilidade na estrutura dos alvos florestais, aliada ainda a disponibilizaçáo de informação dual paralela (HH e VV).

\section{Classificação Context}

Ao se utilizar o classificador Context com o conjunto das quatro componentes $\left(A_{H H}, A_{v v}, A_{<H H . V V^{*}}, A_{\text {entropia }}\right)$, foi alcançado um desempenho categorizado como "muito bom", decorrente de um elevado valor de Kappa $(0,704)$. Na Figura 5 é apresentada a espacialização das classes temáticas e na Tabela 3 , a respectiva matriz de confusão e valores de concordância associados.

Neste tipo de abordagem contextual, áreas legendadas como pasto sujo apresentaram um fraco desempenho classificatório perante as outras classes investigadas, com somente $59 \%$ dos pixels classificados corretamente; isso em parte se deve a certa similaridade de resposta polarimétrica com a classe capoeira (22\%), caracterizada por constituir-se de um estádio de sucessão inicial, confusão essa já observada na análise das amostras através da distância JM, com valor de 0,317 . Os pares de amostras com maior separabilidade corresponderam aos binômios temáticos "floresta - solo exposto" $(\mathrm{JM}=1,977)$ e "solo exposto - capoeira" $(\mathrm{JM}=$ $1,878)$.

Ao comparar as classificações MAXVER (Kappa $=0,53) \mathrm{e}$ Context $(K a p p a=0,70)$ e em seguida efetuar uma análise do 


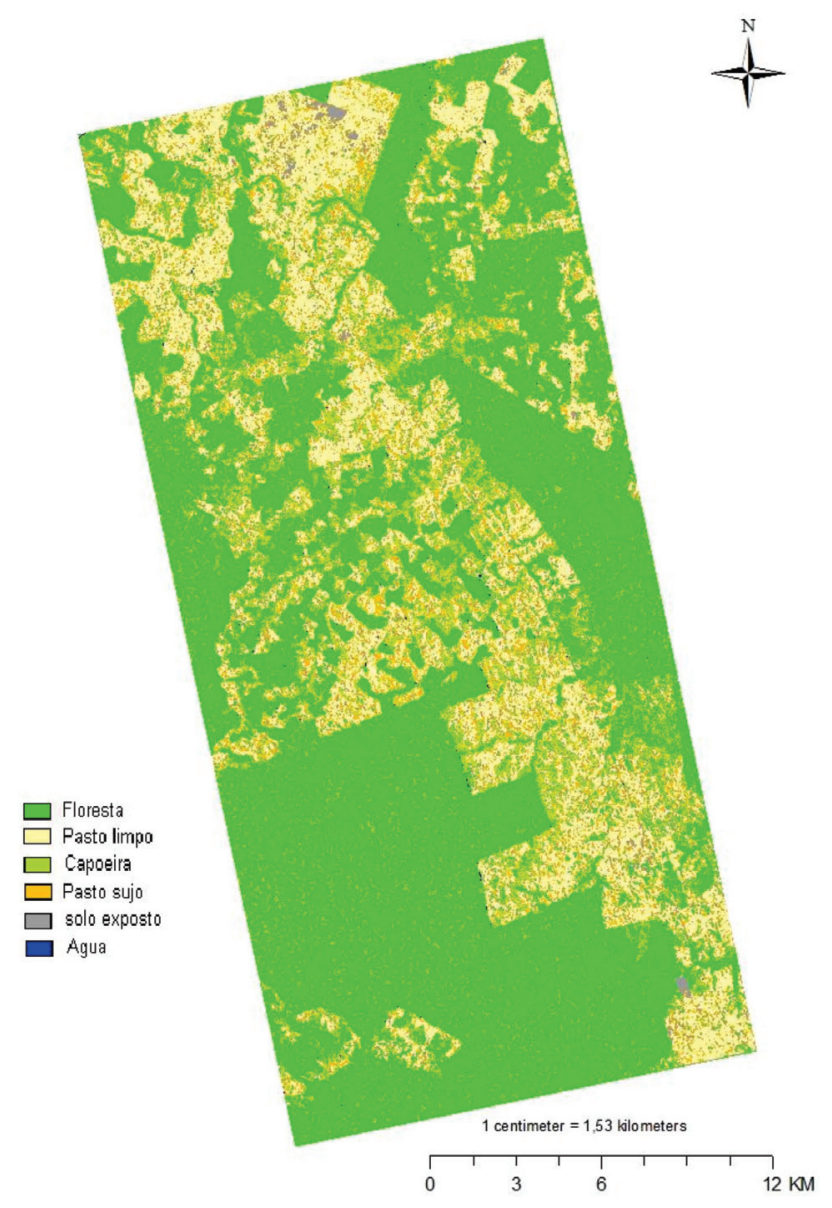

Figura 5 - Classificação temática pelo método Context do conjunto de imagens $\left(A_{H H}, A_{v v^{\prime}} A_{<H H . W^{*}>}, A_{\text {entropia }}\right)$.

kappa condicional para cada classe (Tabela 4), e considerando as informaçôes obtidas nas matrizes de confusão que foram consideradas diferentes de acordo com o teste $Z$, ao nível de significância de $5 \%$, pode-se afirmar que a classificação pelo método Context apresentou melhor desempenho para separar as seis diferentes classes ao se fazer uso das quatro componentes em amplitude da imagem TerraSAR-X

Tabela 3 - Matriz de confusão derivada da classificação pelo método Context.

\begin{tabular}{lcccccc}
\hline & Água & Capoeira & Floresta & $\begin{array}{c}\text { Pasto } \\
\text { limpo }\end{array}$ & $\begin{array}{c}\text { Pasto } \\
\text { sujo }\end{array}$ & $\begin{array}{c}\text { Solo } \\
\text { exposto }\end{array}$ \\
\hline Água & $\mathbf{8 1 , 8 2}$ & 0,00 & 1,15 & 0,00 & 0,00 & 0,00 \\
Capoeira & 0,00 & $\mathbf{1 0 0 , 0 0}$ & 11,49 & 11,63 & 22,73 & 0,00 \\
Floresta & 0,00 & 0,00 & $\mathbf{8 1 , 6 1}$ & 0,00 & 9,09 & 0,00 \\
Pasto Limpo & 0,00 & 0,00 & 1,15 & $\mathbf{7 2 , 0 9}$ & 0,00 & 0,00 \\
\hline Pasto Sujo & 0,00 & 0,00 & 3,45 & 13,95 & $\mathbf{5 9 , 0 9}$ & 0,00 \\
\hline Solo Exposto & 18,18 & 0,00 & 1,15 & 2,33 & 9,09 & $\mathbf{1 0 0 , 0 0}$ \\
\hline
\end{tabular}

Exatidão Global $=78.33 \%$ Kappa $=0.704(\mathrm{Var}=3,42 \mathrm{E}-04)$
Tabela 4 - Valores de Kappa condicional para cada classe derivado das classificações MAXVER e Context.

\begin{tabular}{lcccc}
\hline & \multicolumn{2}{c}{ Maxver } & \multicolumn{2}{c}{ Context } \\
\cline { 2 - 5 } & $\begin{array}{c}\text { Kappa } \\
\text { Condicional }\end{array}$ & $\operatorname{Var}$ (Kappa) & $\begin{array}{c}\text { Kappa } \\
\text { Condicional }\end{array}$ & Var (Kappa) \\
\hline Água & 0,6931 & $3,03 \mathrm{E}-04$ & 0,983 & $2,36 \mathrm{E}-04$ \\
Capoeira & 0,2461 & $1,38 \mathrm{E}-05$ & 0,623 & $1,79 \mathrm{E}-03$ \\
Floresta & 0,9262 & $5,39 \mathrm{E}-06$ & 0,880 & $1,38 \mathrm{E}-03$ \\
Pasto Limpo & 0,6247 & $9,46 \mathrm{E}-06$ & 0,981 & $3,03 \mathrm{E}-04$ \\
Pasto Sujo & 0,2667 & $1,32 \mathrm{E}-05$ & 0,727 & $3,10 \mathrm{E}-03$ \\
Solo Exposto & 0,6523 & $2,33 \mathrm{E}-05$ & 0,718 & $1,76 \mathrm{E}-03$ \\
\hline
\end{tabular}

\section{CONCLUSÕES}

Em função dos procedimentos metodológicos adotados no presente trabalho, foi possível chegar às seguintes conclusões:

A classificação contextual apresentou melhor desempenho na classificaçáo dos dados derivados do TerraSAR-X, apresentando um índice Kappa de 0.70, com uma exatidão de $78.33 \%$ superior àquele do algoritmo classificatório MAXVER.

As polarizaçóes duais $\mathrm{HH}$ eVV, de maneira individualizada, mostraram-se limitadas para separar as diversas classes de uso e cobertura da terra pré-estabelecidas, sendo necessária no conjunto de dados tratados, a inclusão de elementos derivados da matriz de covariância, como a componente $<\mathrm{HH} . \mathrm{VV}^{*}>$ e do parâmetro incoerente "entropia", derivado da decomposição de alvos.

$\mathrm{Na}$ análise exploratória dos dados TerraSAR-X, foi verificado que as classes pasto sujo e capoeira foram as de mais difícil separabilidade. Este fato ficou evidenciado na análise dos gráficos blox-plot, nos valores de distância JM entre classes e confirmados nas matrizes de confusáo das classificaçóes, independente do algoritmo utilizado, se pontual ou contextual.

Numa perspectiva de prosseguimento da linha investigativa, recomenda-se utilizar dados TerraSAR-X full polarimétricos $(\mathrm{HH}, \mathrm{HV}, \mathrm{VH}, \mathrm{VV})$, o que provavelmente permitirá uma maior exploração dos diversos atributos coerentes e incoerentes contidos nas imagens-radar, ampliando inclusive o espectro de abordagens exploratórias (por exemplo, de análise das assinaturas polarimétricas das diversas classes) e de outros algoritmos de classificação. Dados no modo Tanden do TerraSAR-X seriam sobremaneira indicados por incluir as características SAR interferométricas, muito úteis na caracterização estrutural da tipologia florestal em zonas tropicais, de maior complexidade de paisagens.

Em suma, os dados TerraSAR-X investigados possuem certa capacidade de discriminaçáo das classes de uso e cobertura da terra nessa porção da Amazônia brasileira, respeitando-se as limitaçốes pelo modo dual polarimétrico 
(HH e VV) do produto disponível aos usuários, além da freqüência (banda X) inerente ao sistema radar em seu processo de interação com a superfície do alvo.

\section{AGRADECIMENTOS}

Os autores agradecem ao Conselho Nacional de Desenvolvimento Científico e Tecnológico (CNPq) pela concessáo da bolsa do Programa de Estudante-Convênio de Pós-Graduaçáo (PECPG) de pesquisa e de produtividade em pesquisa; ao Dr. Steffen Kuntz, da INFOTERRA, pela cessão de imagens TerraSAR-X e ao Programa Nacional de Cooperação Acadêmica (PROCAD/CAPES) no decorrer da viagem científica à Universidade Federal do Acre (UFAC), dentro do acordo de cooperação INPA/UFRR/UFAC/INPE.

\section{BIBLIOGRAFIA CITADA}

Asner, G.P. 2001. Cloud cover in Landsat observations of the Brazilian Amazon. International Journal of Remote Sensing, 22: 3855-3862.

Cloude, S.R.; Pottier, E.,W. 1996. A review of target decomposition theorems in radar polarimetry. IEEE Transactions. on Geoscience and Remote Sensing, 34(2): 498-518.

Cloude, S.R.; Pottier, E. 1997. An entropy based classification scheme for land application of polarimetric SAR. IEEE Transactions on Geoscience and Remote Sensing, 35(1): 68-78.

Cloude, S.R. 2007. The dual polarization entropy/alpha decomposition: a PALSAR case study. p.19. In: POLinSAR Workshop, June 22-26th, 2007. Frascati, Italy. ESA. Abstract Book.

CPTEC. 2010. Centro de previsão de tempo e estudos climáticos, (http://clima1.cptec.inpe.br/ -rclima1/monitoramento_brasil. shtml). Acesso em 25/07/2010.

Dobson, M.C.; Ulaby, F.T.; Pierce, L.E.; Sharick, T.L.; Bergen, K.M.; Kellndorfer, J.; Kendra, J.R.; Li, E.; Lin, Y.C.; Nashashbi, A.; Sarabandi, K.; Siqueira, P. 1995. Estimation of forest biophysical characteristics in Northern Michigan with SIRC/X-SAR. IEEE Transactions on Geoscience and Remote Sensing, 33: 877-895.

Eineder, M.; Fritz, T.; Mittermayer, J.; Roth, A.; Börner, E.; Breit, H. 2008. TerraSAR-X ground segment - Basic product specification document. In: TerraSAR, Oberpfaffenhofen: DLR - Cluster Applied Remote Sensing, 103 pp.

Freeman, A.; Durden, S.L. 1998. A three-component scattering model for polarimetric SAR data. IEEE Transactions on Geoscience and Remote Sensing, 36(3): 963-973.

Freitas, C.C.; Soler, L.S., Sant'anna S. J. S., Dutra, L.V.; Santos, J.R.; Mura, J.C.; Correia, A.H. 2008. Land Use and Land Cover Mapping in the Brazilian Amazon Using Polarimetric Airborne P-Band SAR Data. IEEE Transactions on Geoscience and Remote Sensing, 46: 2956-2970.

Garcia, C.E. 2011. Análise de imagem polarimétrica TerraSAR-X para classificação de uso e cobertura da terra na porção sudoeste da Amazônia Brasileira. Tese de Mestrado em Sensoriamento
Remoto, Instituto Nacional de Pesquisas Espaciais, São José dos Campos, São Paulo. 84 pp.

Gonçalves, F.G.; Santos, J.R.; Treuhaft, R. N. 2011. Stem volume of tropical forests from polarimetric radar. International Journal of Remote Sensing, 32(2): 503- 522.

Gong, P.; Howarth, P. J. 1992. Frequency-based contextual classification and gray-level vector reduction for land-use identification. Photogrammetric Engineering \& Remote Sensing, 58(4): 423-437.

Guerra, J.B; Mura, J.C; Freitas, C.C. 2010. Discriminação de incrementos de desflorestamento na Amazônia com dados SAR R-99B em banda L. Acta Amazonica, 40: 557-566.

Henderson, F.M.; Lewis, A.J. 1998. Manual of remote sensing: principles and applications of imaging radar, 3rd edition. USA: John Wiley Sons. 896 pp

Hoekman, D.H.; Quiñones, M.J. 2000. Land cover type and biomass classification using AirSAR data for evaluation of monitoring scenarios in the Colombian Amazon. IEEE Transactions on Geoscience and Remote Sensing, 38(2): 685-696.

Houghton, R.A.; Skole, D.L.; Nobre, C.A.; Hackler, J.L.; Lawrence, K.T.; Chomentowski, W. H. 2000. Annual fluxes of carbon from deforestation and regrowth in the Brazilian Amazon. Nature, 403: 301-304.

Kasischke, E.S.; Melack, J.M.; Dobson, C.M. 1997. The use of imaging radars for ecological applications - A review. Remote Sensing of Environment, 59 (2): 141- 156.

Landis, J.R.; Koch, G.G. 1977. The measurements of observer agreement for categorical data. Biometrics, 33: 159-174.

Leckie, D.G., Ranson, K.J. 1998. Forestry applications using imaging radar. cap 9, p. 435-509, In: Henderson, F. M.; Lewis, A. J. (Ed.). Principles and Applications of Imaging Radar. v.2, ed.3., John Wiley. New York.

Lee, J. S.; Grunes, M. R.; De Grandi, G. 1999. Polarimetric SAR speckle filtering and its implication for classification. IEEE Transactions on Geoscience and Remote Sensing. 37(5).

Lee, J.S.; Grunes, M.R.; Pottier, E. 2001. Quantitative comparison of classification capability: fully polarimetric versus dual and single polarization SAR. IEEE Transactions on Geoscience and Remote Sensing, 39(11): 2343-2351.

Lim, H.S.; Matjafri, M.Z.; Abdullah, K.; Saleh, N.M. 2008. Analysis of land covers over Northern peninsular Malaysia by using ALOS-PALSAR data based on frequency-based contextual and neural network classification technique. In: ALOS PI 2008 Symposium.11, ALOS PI 2008 Symposium.

McNairn, H.; Duguay, C.; Brisco, B.; Pultz, T.Z. 2002. The effect of soil and crop residue characteristics on polarimetric radar response. Remote Sensing of Environment, 80(2): 308-320.

Narvaes, I. S. 2010. Avaliação de dados SAR polarimétricos para estimativa de biomassa em diferentes fitofisionomias de florestas tropicais. Tese de Doutorado em Sensoriamento Remoto, Instituto Nacional de Pesquisas Espaciais, São José dos Campos, São Paulo. 164 pp. 
Neeff, T.; Dutra, L.V.; Santos, J.R.; Freitas, C.C.; Araujo, L.S. 2005a. Tropical forest biomass measurement by interferometric height modeling and P-band radar backscatter. Forest Science, 51(6): 585-594.

Neeff, T.; Dutra, L.V.; Santos, J. R.; Freitas, C.C.; Araujo, L.S. 2005b. Power spectrum analysis of SAR data for spatial forest characterization in Amazonia. International Journal of Remote Sensing, 26(13): 2851-2865.

Pitz, W.; Miller, D. 2010. The TerraSAR-X satellite. IEEE Transactions on Geoscience and Remote Sensing 48(2): 615-622.

Pope, K. O.; Benayas-Rey, J. M.; Paris, J. F. 1994. Radar remote sensing of forest and wetland ecosystems in the Central American tropics. Remote Sensing of Environment, 48(2): 205-219.

RADAMBRASIL. 1978. Folhas SC.19 Rio Branco: geologia, geomorfologia, pedologia, vegetação e uso potencial da terra. Ministério das Minas e Energia, Departamento Nacional de Produção Mineral (DNPM), Levantamento de Recursos Naturais, Rio de Janeiro, 554 pp.

Richards, J.A., Jia, X. 1999. Remote sensing digital image analysis, 3rd ed. Berlin: Springer-Verlag, 439 pp.

Rignot, E.; Salas, WA.; Skole, D.L. 1997. Mapping deforestation and secondary growth in Rondonia, Brazil, using imaging radar and Thematic Mapper data. Remote Sensing of Environment, (59): 167-179.

Rodrigues, T.G. 2010. Análise da viabilidade de mapeamento topográfico (escala 1:100.000) na região Amazônica através da integração de informaçôes altimétricas SRTM-3 e planimétrica PALSAR: uma avaliaçấo para a Serra dos Carajás (PA). Dissertação de Mestrado. Instituto Nacional de Pesquisas Espaciais, São José dos Campos. São Paulo. 163 pp.

Saatchi, S.S.; Soares, J.V.; Alves, D.S. 1997. Mapping deforestation and land use in Amazon rainforest by using SIR-C Imagery. Remote Sensing of Environment, 59(2): 191-202 pp

Saatchi, S.S.; Halligan, K.; Despain, D.G.; Crabtree, R.L. 2007. Estimation of forest fuel load from radar remote sensing. IEEE Transactions on Geoscience and Remote Sensing, 45(6): 1726 1740.
Santos, J.R.; La Cruz, M.S.P.; Araújo, L.S. 2002. Savanna and tropical rainforest biomass estimation using JERS-1 data. International Journal of Remote Sensing, 23: 1217-1229.

Santos, J.R.; Mura, J.C.; Paradella, W.R.; Dutra, L.V.; Gonçalves, F.G. 2008. Mapping recent deforestation in the Brazilian Amazon using simulated L-band MAPSAR images. International Journal of Remote Sensing, 29: 4879-4889

Santos, J.R.; Narvaes, I.S.; Graça, P.M.L.A.; Gonçalves, F.G. 2009. Polarimetric responses and scattering mechanisms of tropical forests in the Brazilian Amazon, p. 183-206. In: Gary Jedlovec (NASA/ MSFC-USA). (Org.). Advances on Geoscience and Remote Sensing. 1a. ed. v. 8. Vukovar,Croatia: IN-TECH.

Ulaby, F.T.; Moore, R. K.; Fung, A.K. 1981. Microwave remote sensing: active and passive, vol. I, Microwave remote sensing fundamentals and radiometry, Addison-Wesley, Advanced Book Program, Reading, Massachusetts. 456 pp.

Ulaby, F.T.; M.C. Dobson. 1989. Handbook of Radar Scattering Statistics for Terrain, Artech House, Massachusetts. 362 pp.

Ulaby, F.T.; Elachi, C. 1990. Radar polarimetry for geoscience applications: Artech House, London. UK. 364 pp.

Van Der Sanden, J.J.; Hoekman, D. H. 1999. Potential of airborne radar to support the assessment of land cover in a tropical rain forest environment. Remote Sensing of Environmen, 68: 26-40.

Vieira, I.C.G.; Silva, J.M.C.; Toledo, P.M. Estratégias para evitar a perda biodiversidade na Amazônia. 2005. Estudos Avançados, 19(54): 153-164.

Vieira, P.R. Desenvolvimento de classificadores de máxima verossimilhança e ICM para imagens SAR, 1996. Dissertaçáo (Mestrado em Sensoriamento Remoto). Instituto Nacional de Pesquisas Espaciais, São José dos Campos. 251 pp.

Werninghaus, R.; Buckreuss, S. 2010. The TerraSAR-X mission and system design, IEEE Transactions on Geoscience and Remote Sensing 48(2): 606-614

Recebido em 12/02/2011

Aceito em 18/04/2011 